\title{
Flavor Asymmetry of the Light Quark Sea from Semi-inclusive Deep-Inelastic Scattering
}

K. Ackerstaff, ${ }^{5}$ A. Airapetian, ${ }^{32}$ N. Akopov, ${ }^{32}$ I. Akushevich, ${ }^{6}$ M. Amarian, ${ }^{32,27}$ E. C. Ashenauer,${ }^{13,14}$ H. Avakian, ${ }^{10}$ R. Avakian, ${ }^{32}$ A. Avetissian, ${ }^{32}$ B. Bains, ${ }^{15}$ C. Baumgarten, ${ }^{23}$ M. Beckmann, ${ }^{12}$ St. Belostotski, ${ }^{26}$ J. E. Belz,${ }^{28,29}$ Th. Benisch, ${ }^{8}$ S. Bernreuther, ${ }^{8}$ N. Bianchi, ${ }^{10}$ J. Blouw ${ }^{25}$ H. Böttcher, ${ }^{6}$ A. Borissov, ${ }^{6,14}$ J. Brack, ${ }^{4}$ S. Brauksiepe, ${ }^{12}$ B. Braun, ${ }^{8}$ B. Bray, ${ }^{3}$ St. Brons, ${ }^{6}$ W. Brückner, ${ }^{14}$ A. Brülll, ${ }^{14}$ E. E. W. Bruins,${ }^{20}$ H. J. Bulten, ${ }^{18,25,31}$ R. V. Cadman, ${ }^{15}$ G. P. Capitani, ${ }^{10}$ P. Carter, ${ }^{3}$ P. Chumney, ${ }^{24}$ E. Cisbani, ${ }^{27}$ G. R. Court, ${ }^{17}$ P. F. Dalpiaz, ${ }^{9}$ E. De Sanctis,${ }^{10}$

D. De Schepper, ${ }^{20}$ E. Devitsin, ${ }^{22}$ P. K. A. de Witt Huberts, ${ }^{25}$ P. Di Nezza, ${ }^{10}$ M. Düren, ${ }^{8}$ A. Dvoredsky, ${ }^{3}$ G. Elbakian, ${ }^{32}$ A. Fantoni, ${ }^{10}$ A. Fechtchenko, ${ }^{7}$ M. Ferstl,${ }^{8}$ K. Fiedler ${ }^{8}$ B. W. Filippone,${ }^{3}$ H. Fischer, ${ }^{12}$ B. Fox, ${ }^{4}$ J. Franz, ${ }^{12}$ S. Frullani, ${ }^{27}$ M.-A. Funk, ${ }^{5}$ N. D. Gagunashvili, ${ }^{7}$ H. Gao, ${ }^{2,15}$ Y. Gärber, ${ }^{6}$ F. Garibaldi, ${ }^{27}$ G. Gavrilov, ${ }^{26}$ P. Geiger, ${ }^{14}$ V. Gharibyan, ${ }^{32}$ V. Giordjian, ${ }^{10}$ A. Golendukhin, ${ }^{19,32}$ G. Graw,${ }^{23}$ O. Grebeniouk, ${ }^{26}$ P. W. Green, ${ }^{1,29}$ L. G. Greeniaus, ${ }^{1,29}$ C. Grosshauser, ${ }^{8}$ A. Gute, ${ }^{8}$ W. Haeberli, ${ }^{18}$ J.-O. Hansen, ${ }^{2}$ D. Hasch,${ }^{6}$ O. Häusser, ${ }^{28,29, *}$ R. Henderson, ${ }^{29}$ Th. Henkes,${ }^{25}$ M. Henoch, ${ }^{8}$ R. Hertenberger, ${ }^{23}$ Y. Holler, ${ }^{5}$ R. J. Holt, ${ }^{15}$ W. Hoprich,${ }^{14}$ H. Ihssen,,${ }^{5,25}$ M. Iodice, ${ }^{27}$ A. Izotov, ${ }^{26}$ H. E. Jackson, ${ }^{2}$ A. Jgoun, ${ }^{26}$ R. Kaiser, ${ }^{28,29}$ E. Kinney, ${ }^{4}$ A. Kisselev, ${ }^{26}$ P. Kitching, ${ }^{1}$ H. Kobayashi,${ }^{30}$ N. Koch, ${ }^{19}$ K. Königsmann, ${ }^{12}$ M. Kolstein, ${ }^{25}$ H. Kolster, ${ }^{23}$ V. Korotkov, ${ }^{6}$ W. Korsch, ${ }^{3,16}$ V. Kozlov, ${ }^{22}$ L. H. Kramer, ${ }^{20,11}$ V. G. Krivokhijine, ${ }^{7}$ M. Kurisuno,${ }^{30}$ G. Kyle,${ }^{24}$ W. Lachnit, ${ }^{8}$ W. Lorenzon, ${ }^{21}$ N. C. R. Makins, ${ }^{2,15}$ S. I. Manaenkov, ${ }^{26}$ F. K. Martens, ${ }^{1}$ J. W. Martin, ${ }^{20}$ F. Masoli, ${ }^{9}$ A. Mateos,${ }^{20}$ M. McAndrew,${ }^{17}$ K. McIlhany, ${ }^{3}$ R. D. McKeown,${ }^{3}$ F. M. Menden, ${ }^{29}$ F. Meissner, ${ }^{6}$ A. Metz,${ }^{23}$ N. Meyners,${ }^{5}$ O. Mikloukho, ${ }^{26}$ C. A. Miller, ${ }^{1,29}$ M. A. Miller, ${ }^{15}$ R. Milner, ${ }^{20}$ V. Mitsyn, ${ }^{7}$ A. Most,${ }^{15,21}$ R. Mozzetti, ${ }^{10}$ V. Muccifora,,${ }^{10}$ A. Nagaitsev,${ }^{7}$ Y. Naryshkin, ${ }^{26}$ A. M. Nathan, ${ }^{15}$

F. Neunreither, ${ }^{8}$ M. Niczyporuk, ${ }^{20}$ W.-D. Nowak, ${ }^{6}$ M. Nupieri, ${ }^{10}$ T. G. O’Neill, ${ }^{2}$ J. Ouyang, ${ }^{29}$ B. R. Owen, ${ }^{15}$ V. Papavassiliou ${ }^{24}$ S. F. Pate,${ }^{20,24}$ M. Pitt, ${ }^{3}$ S. Potashov,${ }^{22}$ D. H. Potterveld,${ }^{2}$ G. Rakness,${ }^{4}$ A. Reali, ${ }^{9}$ R. Redwine,${ }^{20}$ A. R. Reolon, ${ }^{10}$ R. Ristinen, ${ }^{4}$ K. Rith, ${ }^{8}$ H. Roloff, ${ }^{6}$ P. Rossi, ${ }^{10}$ S. Rudnitsky, ${ }^{21}$ M. Ruh, ${ }^{12}$ D. Ryckbosch, ${ }^{13}$ Y. Sakemi, ${ }^{30}$ I. Savin, ${ }^{7}$ F. Schmidt,${ }^{8}$ H. Schmitt, ${ }^{12}$ G. Schnell, ${ }^{24}$ K. P. Schüler, ${ }^{5}$ A. Schwind, ${ }^{6}$ T.-A. Shibata, ${ }^{30}$ K. Shibatani, ${ }^{30}$ T. Shin, ${ }^{20}$ V. Shutov, ${ }^{7}$ C. Simani, ${ }^{9}$ A. Simon, ${ }^{12}$ K. Sinram, ${ }^{5}$ P. Slavich, ${ }^{9}, 10$ M. Spengos, ${ }^{5}$ E. Steffens,${ }^{8}$ J. Stenger, ${ }^{8}$ J. Stewart, ${ }^{17}$ U. Stoesslein, ${ }^{6}$ M. Sutter,${ }^{20}$ H. Tallini,,${ }^{17}$ S. Taroian, ${ }^{32}$ A. Terkulov,${ }^{22}$ B. Tipton,,${ }^{20}$ M. Tytgat, ${ }^{13}$ G. M. Urciuoli, ${ }^{27}$ J. J. van Hunen, ${ }^{25}$ R. van de Vyver, ${ }^{13}$ J. F. J. van den Brand, ${ }^{25,31}$ G. van der Steenhoven, ${ }^{25}$ M. C. Vetterli, ${ }^{28,29}$ M. G. Vincter, ${ }^{29}$ E. Volk, ${ }^{14}$ W. Wander, ${ }^{8}$ S. E. Williamson, ${ }^{15}$ T. Wise, ${ }^{18}$ K. Woller, ${ }^{5}$ S. Yoneyama, ${ }^{30}$ and $\mathrm{H}$. Zohrabian ${ }^{32}$

(HERMES Collaboration)

${ }^{1}$ Department of Physics, University of Alberta, Edmonton, Alberta, Canada T6G $2 \mathrm{JI}$

${ }^{2}$ Physics Division, Argonne National Laboratory, Argonne, Illinois 60439

${ }^{3}$ W. K. Kellogg Radiation Lab, California Institute of Technology, Pasadena, California 91125

${ }^{4}$ Nuclear Physics Laboratory, University of Colorado, Boulder, Colorado 80309-0446

${ }^{5}$ DESY, Deutsches Electronen Synchrotron, 22603 Hamburg, Germany

${ }^{6}$ DESY, 15738 Zeuthen, Germany

${ }^{7}$ Joint Institute for Nuclear Research, 141980 Dubna, Russia

${ }^{8}$ Physikalisches Institut, Universität Erlangen-Nürnberg, 91058 Erlangen, Germany

${ }^{9}$ Dipartimento di Fisica, Università di Ferrara, 44100 Ferrara, Italy

${ }^{10}$ Istituto Nazionale di Fisica Nucleare, Laboratori Nazionali di Frascati, O0044 Frascati, Italy

${ }^{11}$ Department of Physics, Florida International University, Miami, Florida 33199

${ }^{12}$ Fakultaet fuer Physik, Universität Freiburg, 79104 Freiburg, Germany

${ }^{13}$ Department of Subatomic and Radiation Physics, University of Gent, 9000 Gent, Belgium

${ }^{14}$ Max-Planck-Institut für Kernphysik, 69029 Heidelberg, Germany

${ }^{15}$ Department of Physics, University of Illinois, Urbana, Illinois 61801

${ }^{16}$ Department of Physics and Astronomy, University of Kentucky, Lexington, Kentucky 40506

${ }^{17}$ Physics Department, University of Liverpool, Liverpool L69 7ZE, United Kingdom

${ }^{18}$ Department of Physics, University of Wisconsin-Madison, Madison, Wisconsin 53706

${ }^{19}$ Physikalisches Institut, Philipps-Universität Marburg, 35037 Marburg, Germany

${ }^{20}$ Laboratory for Nuclear Science, Massachusetts Institute of Technology, Cambridge, Massachusetts 02139

${ }^{21}$ Randall Laboratory of Physics, University of Michigan, Ann Arbor, Michigan 48109-1120

${ }^{22}$ Lebedev Physical Institute, 117924 Moscow, Russia

${ }^{23}$ Sektion Physik, Universität München, 85748 Garching, Germany

${ }^{24}$ Department of Physics, New Mexico State University, Las Cruces, New Mexico 88003

${ }^{25}$ Nationaal Instituut voor Kernfysica en Hoge-Energiefysica (NIKHEF), 1009 DB Amsterdam, The Netherlands

${ }^{26}$ Petersburg Nuclear Physics Institute, St. Petersburg, 188350 Russia 


\section{${ }^{27}$ Istituto Nazionale di Fisica Nucleare, Sezione Sanitá and Istituto Superiore di Sanitá, Physics Laboratory, 00161 Roma, Italy \\ ${ }^{28}$ Department of Physics, Simon Fraser University, Burnaby, British Columbia, Canada V5A 1S6 \\ ${ }^{29}$ TRIUMF, Vancouver, British Columbia, Canada V6T $2 A 3$ \\ ${ }^{30}$ Tokyo Institute of Technology, Tokyo 152, Japan \\ ${ }^{31}$ Department of Physics and Astronomy, Vrije Universiteit, 1081 HV Amsterdam, The Netherlands \\ ${ }^{32}$ Yerevan Physics Institute, 375036, Yerevan, Armenia}

(Received 15 July 1998; revised manuscript received 12 October 1998)

The flavor asymmetry of the light quark sea of the nucleon is determined in the kinematic range $0.02<x<0.3$ and $1<Q^{2}<10 \mathrm{GeV}^{2}$ for the first time from semi-inclusive deep-inelastic scattering. The quantity $[\bar{d}(x)-\bar{u}(x)] /[u(x)-d(x)]$ is derived from a relationship between the yields of positive and negative pions from unpolarized hydrogen and deuterium targets. The flavor asymmetry $\bar{d}-\bar{u}$ is found to be nonzero and $x$ dependent, showing an excess of $\bar{d}$ over $\bar{u}$ quarks in the proton. [S0031-9007(98)08024-7]

PACS numbers: 13.60.Hb, 11.30.Hv, 14.20.Dh, 25.30.Fj

The flavor content of the nucleon sea has come to be recognized as an important domain for testing models of nucleon structure [1]. Until recently there has been little experimental constraint on the flavor asymmetry in the quark distributions of the light quark sea in the nucleon. The first was an integral test, based on a comparison of inclusive deep-inelastic scattering on the proton and the neutron to determine the Gottfried sum [2] defined as

$$
S_{G} \equiv \int_{0}^{1} \frac{d x}{x}\left[F_{2}^{p}(x)-F_{2}^{n}(x)\right],
$$

where $F_{2}^{p}(x)$ and $F_{2}^{n}(x)$ are the structure functions of the proton and neutron, respectively. The assumption of isospin symmetry in the quark-proton model allows the sum to be written as

$$
\begin{aligned}
S_{G}= & \frac{1}{3} \int_{0}^{1}\left[u_{v}(x)-d_{v}(x)\right] d x \\
& -\frac{2}{3} \int_{0}^{1}[\bar{d}(x)-\bar{u}(x)] d x,
\end{aligned}
$$

where $u_{v}(x)$ and $d_{v}(x)$ are the density functions of the valence quarks and $\bar{u}(x)$ and $\bar{d}(x)$ those of the antiquarks in the proton, and $x$ is the fraction of the nucleon light cone momentum carried by the struck quark. A flavor symmetric sea, $\bar{u}(x)=\bar{d}(x)$, leads to the Gottfried sum rule $S_{G}=1 / 3$. A measurement of the Gottfried sum by NMC [3] resulted in $S_{G}=0.235 \pm 0.026$, at $Q^{2}=$ $4 \mathrm{GeV}^{2}$. If isospin symmetry holds, a global flavor asymmetry $\int_{0}^{1}[\bar{d}(x)-\bar{u}(x)] d x \approx 0.15$ would account for the NMC result. Many ideas such as Pauli blocking and pion clouds have been proposed to explain such a flavor asymmetry in the sea [1]. Two methods have been proposed to measure its $x$ dependence: the Drell-Yan process [4] and semi-inclusive deep-inelastic scattering [5].

Results are presented here for the $x$ dependence of $(\bar{d}-\bar{u}) /(u-d)$ from the analysis of charged pion yields in semi-inclusive deep-inelastic scattering of positrons on unpolarized hydrogen and deuterium targets. The data cover the kinematic range $0.02<x<0.3$ and $1<Q^{2}<10 \mathrm{GeV}^{2}$, where $-Q^{2}$ is the four-momentum transfer squared of the exchanged virtual photon and
$x=Q^{2} / 2 M \nu$ is the Bjorken variable with $M$ being the proton mass, $\nu=E-E^{\prime}$ the virtual photon energy, and $E\left(E^{\prime}\right)$ the energy of the incident (scattered) positron.

The ratio $(\bar{d}-\bar{u}) /(u-d)$ is determined from a quantity that combines good sensitivity to the sea asymmetry with minimal sensitivity to instrumental effects. This is the ratio of the differences between charged pion yields for proton and neutron targets [5]:

$$
r(x, z)=\frac{N_{p}^{\pi^{-}}(x, z)-N_{n}^{\pi^{-}}(x, z)}{N_{p}^{\pi^{+}}(x, z)-N_{n}^{\pi^{+}}(x, z)},
$$

where $z=E^{\pi} / \nu$ is the fraction of the virtual photon energy carried by the pion and $N^{\pi}(x, z)$ is the yield of pions coming from deep-inelastic scattering off nucleons. Factorization between the hard scattering process and the hadronization of the struck quark implies

$$
N^{\pi^{ \pm}}(x, z) \propto \sum_{i} e_{i}^{2}\left[q_{i}(x) D_{q_{i}}^{\pi^{ \pm}}(z)+\bar{q}_{i}(x) D_{\bar{q}_{i}}^{\pi^{ \pm}}(z)\right],
$$

where $e_{i}$ is the quark charge in units of the elementary charge, $q_{i}(x)$ and $\bar{q}_{i}(x)$ are the density distributions of quarks and antiquarks of flavor $i$, and the fragmentation functions $D_{q_{i}}^{\pi^{ \pm}}(z)$ represent the probability that the quark of flavor $i$ fragments to a charged pion. Assuming isospin symmetry between protons and neutrons as well as charge conjugation invariance, the number of light quark fragmentation functions is reduced to two: the favored and disfavored fragmentation functions [6], e.g., $D_{u}^{\pi^{+}}$and $D_{u}^{\pi^{-}}$. Using Eq. (4) to express $r(x, z)$ in terms of quark distributions and fragmentation functions results in

$$
\frac{1+r(x, z)}{1-r(x, z)}=\frac{u(x)-d(x)+\bar{u}(x)-\bar{d}(x)}{[u(x)-\bar{u}(x)]-[d(x)-\bar{d}(x)]} J(z),
$$

where $J(z)=\frac{3}{5}\left(\frac{1+D^{\prime}(z)}{1-D^{\prime}(z)}\right)$ and $D^{\prime}(z)=D_{u}^{\pi^{-}}(z) / D_{u}^{\pi^{+}}(z)$. It should be noted that the right-hand side of Eq. (5) factorizes into two independent functions of $x$ and $z$, respectively. Thus, the equation may be rearranged to isolate a quantity sensitive to the flavor asymmetry: 


$$
\frac{\bar{d}(x)-\bar{u}(x)}{u(x)-d(x)}=\frac{J(z)[1-r(x, z)]-[1+r(x, z)]}{J(z)[1-r(x, z)]+[1+r(x, z)]} .
$$

The assumptions leading to Eq. (5) imply that the expression on the right-hand side of Eq. (6) is independent of $z$.

In the HERMES experiment, $27.5 \mathrm{GeV}$ positrons circulating the HERA storage ring at DESY are scattered on internal hydrogen $\left(\mathrm{H}_{2}\right)$, deuterium $\left(\mathrm{D}_{2}\right)$, and helium-3 $\left({ }^{3} \mathrm{He}\right)$ targets. The target gas is fed into the center of a 40-cm-long open-ended storage cell. Systematic uncertainties are minimized by cycling the three gases through one of various permutations, several times during each 8-12 hour HERA fill. The unpolarized luminosity of $(1-5) \times 10^{32} \mathrm{~cm}^{-2} \mathrm{~s}^{-1}$ is monitored by a pair of $\mathrm{NaBi}\left(\mathrm{WO}_{4}\right)_{2}$ electromagnetic calorimeters that detect Bhabha scattering from target electrons.

A detailed description of the HERMES spectrometer is provided elsewhere [7]. It is a forward spectrometer that identifies the scattered positron as well as the associated hadrons, in the scattering angle range of $0.04<$ $\theta<0.22 \mathrm{rad}$. Positron-hadron discrimination is based on information from four particle identification detectors: a threshold gas Čerenkov counter, a transition-radiation detector, a lead-glass electromagnetic calorimeter, and a preshower detector located directly before the calorimeter. This combination provides an average position identification efficiency of $99 \%$, with a hadron contamination that is dependent on the kinematics of the positron but is always less than $1 \%$. One of the features of the spectrometer central to this work is its threshold Čerenkov counter, which distinguishes pions from heavier hadrons for particles with momenta greater than $3.8 \mathrm{GeV}$ while maintaining better than $95 \%$ pion identification efficiency at momenta larger than $6 \mathrm{GeV}$.

The kinematic requirements used in this analysis are $Q^{2}>1 \mathrm{GeV}^{2}$, invariant mass $W$ of the initial photonnucleon system greater than $2 \mathrm{GeV}$, and fractional energy $y=\nu / E$ of the incident lepton transferred to the virtual photon less than 0.85 . Events are required to originate from the target-beam interaction region. For the determination of $r(x, z)$, the data are partitioned in five bins of $x$ in the range $0.02<x<0.3$ and up to six bins in $z$ in the range $0.2<z<0.8$. The pions are selected to be in the current fragmentation region (i.e., derived from the struck quark and not target fragments) by requiring $x_{F}=2 P_{L} / \mathrm{W}$ to be greater than 0.25 , where $P_{L}$ is the momentum component of the pion in the longitudinal direction with respect to the virtual photon in the photon-nucleon center-of-mass frame. Data at Bjorken $x>0.3$ are excluded because they are dominated by scattering from the valence quarks. The yields for the neutron target appearing in Eq. (3) can be inferred from yields obtained from deuterium and hydrogen targets, $N_{n}^{\pi^{ \pm}}=N_{d}^{\pi^{ \pm}}-N_{p}^{\pi^{ \pm}}$. Thus the determination of $[\bar{d}(x)-\bar{u}(x)] /[u(x)-d(x)]$ is made by taking ratios of differences between yields from hydrogen and deuterium. The pion yields extracted from the data are $N_{\mathrm{det}}^{\pi}(x, z) / \mathcal{L}$, where $N_{\text {det }}^{\pi}(x, z)$ is the number of detected pions associated with deep-inelastic scattering events and $\mathcal{L}$ is the integrated luminosity $\left[\left(\right.\right.$ nuclei $\left.\left./ \mathrm{cm}^{2}\right) / \mathrm{s}\right]$ corrected for instrumental dead time and inefficiencies; the possible effect of different acceptances for $\pi^{+}$and $\pi^{-}$is included in the systematic uncertainties discussed below. The fragmentation functions $D_{q_{i}}^{\pi^{ \pm}}(z)$ and their ratio $D^{\prime}(z)$ were extracted from the measured pion yields from each of the unpolarized targets: $\mathrm{H}_{2}, \mathrm{D}_{2}$, and ${ }^{3} \mathrm{He}$ [8], using Eq. (4) together with the GRV 94 LO [9] parametrization of parton distributions. No statistically significant differences were found between the results from the different targets. To avoid statistical correlations with the pion ratios, only $D^{\prime}(z)$ extracted from ${ }^{3} \mathrm{He}$ is used in this analysis. The fragmentation function ratio $D^{\prime}(z)$ determined within the HERMES acceptance is found to vary with $x$ and $Q^{2}$ typically by less than $10 \%$, except in the low-yield region near $z=0.8$ where as much as $40 \%$ may be seen. However, extraction of the flavor asymmetry in bins of $x$ and $Q^{2}$ revealed the averaged result to be unaffected by this variation. The dependence of the left-right instrumental asymmetry on pion charge is significant only at the lowest momenta and is taken into account as a correction. For all $x$ bins, the resulting change in the sea asymmetry is less than $1.5 \%$.

The results for the quantity $(\bar{d}-\bar{u}) /(u-d)$ are shown in Fig. 1 as a function of $z$ for five bins in $x$. The data show no $z$ dependence and are consistent with the form of factorization shown in Eq. (5). The values of $(\bar{d}-\bar{u}) /(u-d)$ averaged over $z$ are plotted in Fig. 2(a)

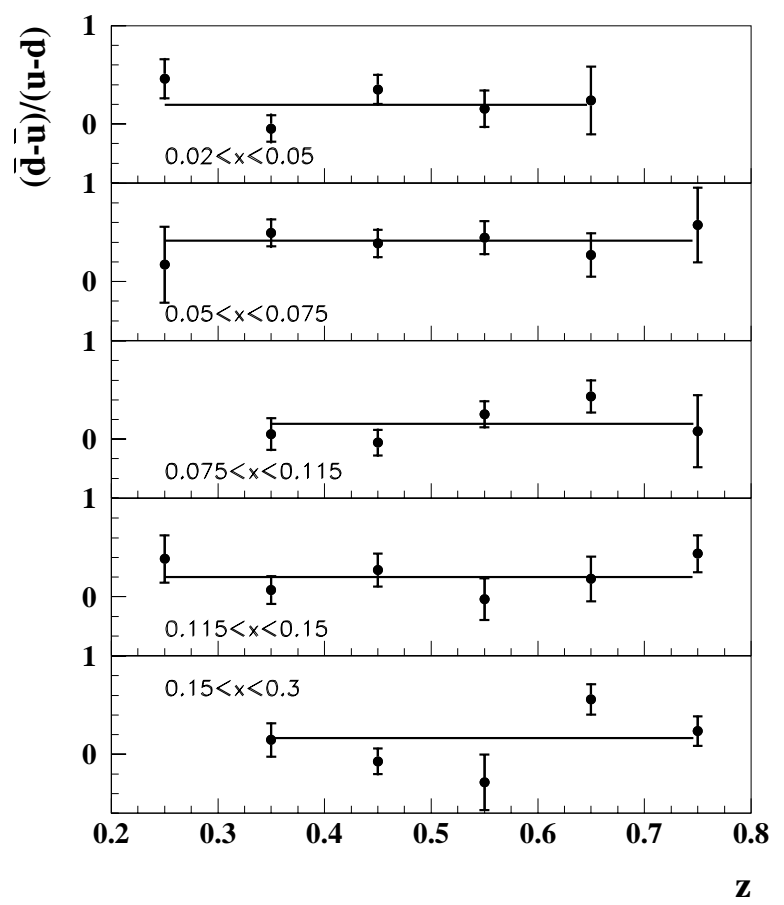

FIG. 1. The distribution $(\bar{d}-\bar{u}) /(u-d)$ as a function of $z$ in five bins of $x$. The points are fit to a constant for each $x$ bin. The error bars represent statistical and systematic uncertainties added in quadrature. Some points are omitted due to limited statistics. 


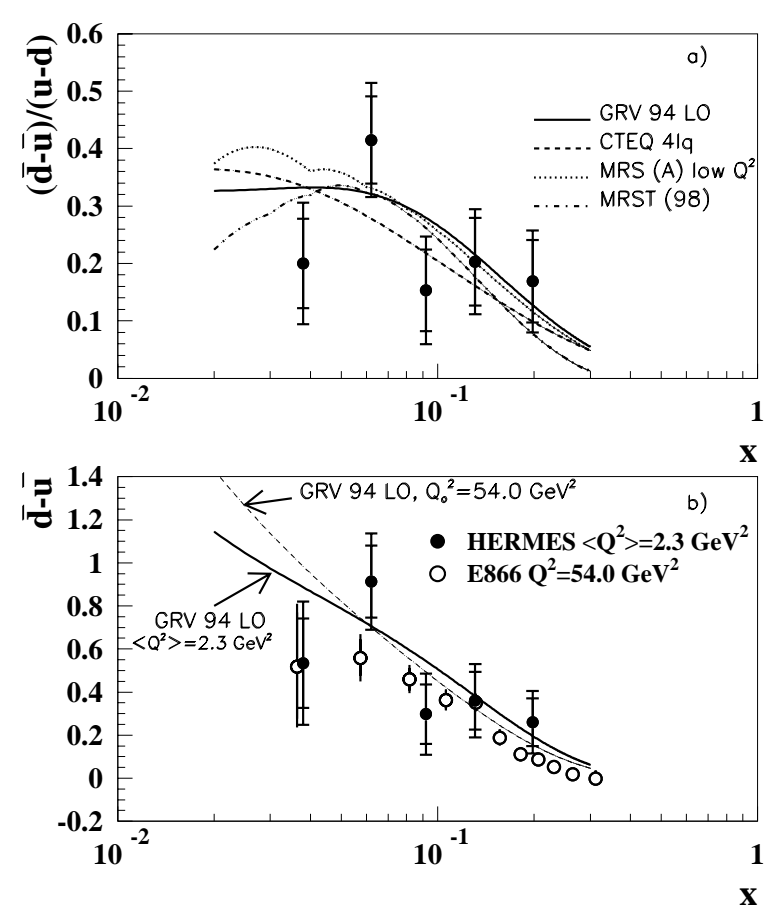

FIG. 2. (a) $(\bar{d}-\bar{u}) /(u-d)$ as a function of $x$. Also included are the GRV 94 LO [9], CTEQ 4lq [10], MRS (A) [11] low $Q^{2}$, and MRST (98) [12] parametrizations calculated at the appropriate $Q^{2}$ for each $x$ bin. (b) $\bar{d}-\bar{u}$ as a function of $x$. The open circles represent the E866 [15] determination of $\bar{d}-\bar{u}$ at $Q_{0}^{2}=54 \mathrm{GeV}^{2}$. The solid (dashed) curve is the GRV 94 LO [9] parametrization, evaluated at the $Q_{0}^{2}$ values for the HERMES (E866) experiment. The inner error bars represent the statistical uncertainties while for all data including those from E866, the total error bars represent statistical and systematic uncertainties added in quadrature.

as a function of $x$ and are presented in Table I. They are nonzero and positive everywhere in the measured $x$ region, clearly showing an excess of $\bar{d}$ quarks over $\bar{u}$ quarks in the proton. Also included in this figure are the GRV $94 \mathrm{LO}$ [9], CTEQ 4lq [10], MRS (A) [11] low $Q^{2}$, and MRST (98) [12] parametrizations of $(\bar{d}-\bar{u}) /(u-d)$, calculated at the average $Q^{2}$ of the events in each $x$ bin. The HERMES results are determined without making higher order QCD corrections and so only leading order parametrizations should be compared to them. At low $Q^{2}$, only GRV 94 is available at leading order. The distribution $\bar{d}-\bar{u}$ as a function of $x$ is thus derived from $(\bar{d}-\bar{u}) /(u-d)$ using the GRV 94 LO parametrization of $u(x)-d(x)$, again calculated at the appropriate $Q^{2}$ for each $x$ bin. Other combinations from GRV 94 LO could have been used instead, such as $u_{v}-d_{v}$ or $u+\bar{u}-d-\bar{d}$, but these choices result in more sensitivity to this model input, as indicated by differences seen from substituting the GRV 98 LO parametrization [13]. The results are presented in Fig. 2(b) and Table I and give an integral over the measured $x$ region of $\int_{0.02}^{0.3}[\bar{d}(x)-\bar{u}(x)] d x=0.107 \pm$ 0.021 (stat) \pm 0.017 (syst), after the integrand at each $x$ bin is "evolved" to the average measured $Q_{0}^{2}$ of $2.3 \mathrm{GeV}^{2}$ by applying the ratio of the GRV 94 values for $\bar{d}-\bar{u}$ at the two values of $Q^{2}$. The GRV $94 \mathrm{LO}$ parametrization is used to estimate the portion of the integral in the unmeasured regions. The contribution from $x<0.02$ is significant: 0.050 (almost $50 \%$ of that over the measured $x$ range), while the high $x$ region $x>0.3$ contributes only 0.006 . Including an extrapolation uncertainty estimate based on differences among the available parametrizations, the total integral over all $x$ is then approximately $0.16 \pm 0.03$, which is consistent with the results first seen by NMC [3].

An alternate approach to the measurement of the flavor asymmetry is the comparison of the Drell-Yan process on protons and deuterons, which is sensitive to the quantity $\bar{u} / \bar{d}$ [4]. Using this approach, the NA51 Collaboration at CERN determined that $\bar{u} / \bar{d}=0.51 \pm$ $0.04 \pm 0.05$ at $x=0.18$ and a center-of-mass energy of $\sqrt{s}=29 \mathrm{GeV}$ [14]. The E866 Collaboration at Fermilab recently reported a measurement of the flavor asymmetry in the $x$ range $0.02<x<0.345$ [15]. E866 uses the CTEQ 4M [10] parametrization of $\bar{u}+\bar{d}$ to extract $\bar{d}-$ $\bar{u}$ from the measured quantity $\bar{d} / \bar{u}$. These results given at a $Q_{0}^{2}$ of $54 \mathrm{GeV}^{2}$ are included in Fig. 2(b). This figure demonstrates that the sea asymmetry measured in deepinelastic scattering and in Drell-Yan experiments agree, even though the $Q^{2}$ of the two experiments differ by a factor of about 20 .

The total systematic uncertainties associated with the determination of the quantity $(\bar{d}-\bar{u}) /(u-d)$ in the present work are given in Table I. These uncertainties include various effects as follows. After the correction at low momenta, any residual charge-dependent instrumental acceptance asymmetry is estimated to be less than $1.5 \%$. Effects of the finite resolution of the spectrometer and bremsstrahlung have been investigated with Monte Carlo studies. These effects are small and are included in the systematic uncertainty. Radiative corrections to the

TABLE I. Values for $(\bar{d}-\bar{u}) /(u-d)$ and $\bar{d}-\bar{u}$.

\begin{tabular}{ccccccccc}
\hline \hline $\begin{array}{c}x \\
\text { range }\end{array}$ & $\langle x\rangle$ & $\begin{array}{c}\left\langle Q^{2}\right\rangle \\
\left(\mathrm{GeV}^{2}\right)\end{array}$ & $\begin{array}{c}\frac{\bar{d}-\bar{u}}{u-d} \\
\text { error }\end{array}$ & $\begin{array}{c}\text { Syst } \\
\text { error }\end{array}$ & $\bar{d}-\bar{u}$ & $\begin{array}{c}\text { Stat } \\
\text { error }\end{array}$ & $\begin{array}{c}\text { Syst } \\
\text { error }\end{array}$ \\
\hline $0.020-0.050$ & 0.038 & 1.33 & 0.20 & 0.08 & 0.07 & 0.53 & 0.21 & 0.20 \\
$0.050-0.075$ & 0.062 & 1.82 & 0.42 & 0.08 & 0.06 & 0.91 & 0.17 & 0.15 \\
$0.075-0.115$ & 0.092 & 2.52 & 0.15 & 0.07 & 0.06 & 0.30 & 0.14 & 0.13 \\
$0.115-0.150$ & 0.131 & 3.38 & 0.20 & 0.08 & 0.05 & 0.36 & 0.13 & 0.10 \\
$0.150-0.300$ & 0.198 & 4.88 & 0.17 & 0.07 & 0.05 & 0.26 & 0.11 & 0.09 \\
\hline \hline
\end{tabular}


pion yields have been calculated following the prescription of Ref. [16]. Their effect on the pion ratio in Eq. (3) is estimated to be less than $1 \%$ and is included in the systematic uncertainty. Lepton contamination in the pion sample is found to be at most of order $1 \%$, and the differences between the two targets were no more than $0.2 \%$. The effects of hadrons misidentified as pions have been estimated from the Monte Carlo simulations and are also included. Finally, the uncertainty in the fragmentation function ratio $D^{\prime}(z)$ as measured at HERMES has been accounted for. The statistical uncertainty of $D^{\prime}(z)$ has been included in quadrature to the statistical uncertainty of the pion yields. The systematic component of this ratio determined from systematic studies of the HERMES data has been included in quadrature to the total uncertainty.

The only additional systematic uncertainty associated with $\bar{d}-\bar{u}$ is the choice of parametrization to extract $\bar{d}-\bar{u}$ from $(\bar{d}-\bar{u}) /(u-d)$. The GRV 94 LO [9] parametrization of $u(x)-d(x)$ is used. The MRS (A) [11] low $Q^{2}$ parametrization is used as an alternative. The resulting different in the value of $\bar{d}-\bar{u}$ is used as an estimate of this uncertainty.

In summary, the flavor asymmetry of the light quark sea is extracted for the first time from pion yields in semiinclusive deep-inelastic scattering. The data are recorded at the HERMES experiment in the kinematic range of $0.02<x<0.3$ and $1<Q^{2}<10 \mathrm{GeV}^{2}$. The measured quantity $(\bar{d}-\bar{u}) /(u-d)$ is found to be nonzero over the entire $x$ range measured, clearly showing an excess of $\bar{d}$ quarks over $\bar{u}$ quarks in the proton sea. Its $x$ dependence will serve as a further constraint on global parametrizations of parton density functions. The integral over the measured region of the derived quantity $\bar{d}-\bar{u}$ is $\int_{0.02}^{0.3}[\bar{d}(x)-$ $\bar{u}(x)] d x=0.107 \pm 0.021$ (stat) \pm 0.017 (syst) at a $Q_{0}^{2}$ of $2.3 \mathrm{GeV}^{2}$, which accounts for two-thirds of the Gottfried sum rule deficit.

We gratefully acknowledge the DESY management for its support and the DESY staff and the staffs of the collaborating institutions. This work was supported by the FWO-Flanders, Belgium; the Natural Sciences and Engineering Research Council of Canada; the INTAS, $\mathrm{HCM}$, and TMR network contributions from the Eu- ropean Community; the German Bundesministerium für Bildung, Wissenschaft, Forschung und Technologie; the Deutscher Akademischer Austauschdienst (DAAD); the Italian Istituto Nazionale di Fisica Nucleare (INFN); Monbusho, JSPS, and Toray Science Foundation of Japan; the Dutch Foundation for Fundamenteel Onderzoek der Materie (FOM); the U.K. Particle Physics and Astronomy Research Council; and the U.S. Department of Energy and National Science Foundation.

*Deceased.

[1] For a recent review, see F. M. Steffens and A. W. Thomas, Phys. Rev. C 55, 900 (1997), and references therein.

[2] K. Gottfried, Phys. Rev. Lett. 18, 1174 (1967).

[3] NMC Collaboration, M. Arneodo et al., Phys. Rev. D 50, 1 (1994).

[4] S. D. Ellis and W. J. Stirling, Phys. Lett. B 256, 258 (1991).

[5] J. Levelt et al., Phys. Lett. B 263, 498 (1991).

[6] EMC Collaboration, M. Arneodo et al., Nucl. Phys. B321, 541 (1989).

[7] HERMES Collaboration, K. Ackerstaff et al., Nucl. Instrum. Methods Phys. Res., Sect. A 417, 230 (1998).

[8] P. Geiger, Ph.D. thesis, Ruprecht-Karls-Universität, Heidelberg 1998, http://dxhra1.desy.de/notes/pub/98-LIB/ geiger.98.005.ps.gz.

[9] M. Glück et al., Z. Phys. C 67, 433 (1995).

[10] H. L. Lai et al., Phys. Rev. D 55, 1280 (1997).

[11] A. D. Martin et al., Phys. Rev. D 51, 4756 (1995).

[12] A. D. Martin et al., hep-ph/9803445.

[13] M. Glück et al., Dortmund University Report No. DOTH-98-07, hep-ph/9806404, 1998.

[14] NA51 Collaboration, A. Baldit et al., Phys. Lett. B 332, 244 (1994).

[15] E866 Collaboration, E. A. Hawker et al., Phys. Rev. Lett. 80, 3715 (1998); E866 Collaboration, J.C. Peng et al., hep-ph/9804288.

[16] T. V. Kukhto and N. M. Shumeiko, Nucl. Phys. B219, 412 (1983); I. V. Akushevich and N. M. Shumeiko, J. Phys. G 20, 513 (1994); A. V. Soroko and N. M. Shumeiko, in Workshop on Radiative Corrections Relevant for the HERMES Experiment, edited by H. Boettcher and W.-D. Nowak [Report No. DESY-Zeuthen 94-02]. 